\title{
Invariants to convolution with circularly symmetric PSF
}

\author{
Jan Flusser and Barbara Zitová \\ Institute of Information Theory and Automation \\ Academy of Sciences of the Czech Republic \\ Pod vodárenskou věží 4, 18208 Prague 8, Czech Republic \\ \{flusser, zitova\}@utia.cas.cz
}

\begin{abstract}
We introduce a new class of moment-based features invariant to rotation and to convolution with an unknown point-spread function having circular symmetry. Unlike the invariants published earlier, they comprise both even and odd order moments, which increases their discrimination power and robustness. ${ }^{1}$
\end{abstract}

\section{Introduction}

Recognition of objects and patterns that are deformed in various ways has been a goal of much recent research. Degradations (geometric as well as radiometric) are introduced during the image acquisition process by such factors as imaging geometry, illumination changes, wrong focus, lens aberration, systematic and random sensor errors, object occlusion, etc. Finding a set of invariant descriptors is a key step to recognizing degraded objects regardless of the particular deformations.

Large amount of effort has been spent to find appropriate invariants with respect to deformation of spatial coordinates, like rigid-body, affine, and projective transforms. Moment invariants, Fourier-domain invariants, differential invariants, and point sets invariants belong to the most popular classes of such geometric invariants.

Much less attention has been paid to invariants with respect to changes of the image intensity function (we call them radiometric invariants) and to combined radiometricgeometric invariants. In fact, just the invariants both to radiometric and geometric image degradations are necessary to resolve practical object recognition tasks because both types of degradations use to be present together in the input images.

1 This work has been supported by the grants No. 102/01/PO65 and 102/04/0155 of the Grant Agency of the Czech Republic.
An important class of radiometric degradations we are faced with often in practice is image blurring. Blurring can be caused by camera out-of-focus, atmospheric turbulence, vibrations, sensor and/or scene motion, and by interpolation-based enlargement of the image. If the scene is flat and the imaging system is linear and space invariant, image blurring can be modelled by a convolution $g(x, y)=$ $(f * h)(x, y)$, where $f$ is an original image, $g$ is an acquired image and $h$ is a point spread function (PSF) of the imaging system. Since in most practical tasks the PSF is unknown, having the invariants to convolution is of prime importance when recognizing objects in a blurred scene. An alternative approach, that would not require convolution invariants, must include blind image deconvolution, which is an ill-posed and practically unsolvable problem.

A pioneer work on this field was done by Flusser and Suk [1] who derived moment-based invariants to convolution with an arbitrary centrosymmetric PSF, where centrosymmetry means $h(x, y)=h(-x,-y)$ for any $(x, y)$. From the geometric point of view, their descriptors were invariant to translation only. Despite of this, the convolution invariants have found numerous practical applications [2], [3], [4]. A significant improvement motivated by a problem of registration of blurred images was made by Flusser and Zitová. They introduced so-called combined blur-rotation invariants [5] and reported their successful usage in satellite image registration and in camera motion estimation.

All convolution and combined invariants in the above cited publications assumed that the blurring PSF is symmetric with respect to the center or to two perpendicular axes. However, majority of the PSF occurring in practice exhibit circular symmetry, i.e. $h(x, y)=h\left(\sqrt{x^{2}+y^{2}}\right) \equiv h(r)$. Since the set of circularly symmetric functions is a "small" subset of the set of all centrosymmetric functions, we may expect that, in addition to existing invariants, there are some (nontrivial) new invariants.

In this paper we derive convolution invariants which have not been described yet. This is a significant step forward because the new invariants comprise both even and 
odd order moments, while all the earlier convolution invariants were composed from odd order moments only. Thanks to this, we get a two-fold benefit - better discriminability (the new invariants are able to distinguish among different centrosymmetric objects, which has been impossible with the earlier invariants) and higher robustness (to obtain the same number of invariants, moments of lower orders than before are sufficient, and lower-order moments are more stable to additive noise).

\section{Mathematical background}

By image function (or image) we understand any 2-D function having bounded support and finite non-zero integral. Complex moment $c_{p q}$ of order $(p+q)$ of image $f(x, y)$ is defined as

$$
c_{p q}=\int_{-\infty}^{\infty} \int_{-\infty}^{\infty}(x+\mathrm{i} y)^{p}(x-\mathrm{i} y)^{q} f(x, y) d x d y,
$$

where $i$ denotes the imaginary unit. In polar coordinates, complex moment definition becomes the form

$$
c_{p q}=\int_{0}^{\infty} \int_{0}^{2 \pi} r^{p+q+1} e^{\mathrm{i}(p-q) \theta} f(r, \theta) d r d \theta .
$$

Now, let $f^{\prime}$ be a rotated version of $f$, i. e. $f^{\prime}(r, \theta)=$ $f(r, \theta+\alpha)$, where $\alpha$ is the angle of rotation, and let $c_{p q}^{\prime}$ denote the complex moments of $f^{\prime}$. Then thanks to (2) it holds

$$
c_{p q}^{\prime}=e^{-\mathrm{i}(p-q) \alpha} \cdot c_{p q} .
$$

Complex moments are affected by convolution in the following way. Let $g(x, y)$ be a filtered version of image $f(x, y)$ with a PSF $h(x, y)$, i.e. $g(x, y)=(f * h)(x, y)$. Then we have

$$
c_{p q}^{(g)}=\sum_{k=0}^{p} \sum_{j=0}^{q}\left(\begin{array}{l}
p \\
k
\end{array}\right)\left(\begin{array}{l}
q \\
j
\end{array}\right) c_{k j}^{(h)} c_{p-k, q-j}^{(f)}
$$

for any $p$ and $q$.

In the following text, we assume that the PSF has circular symmetry, i.e. in polar coordinates $h(r, \theta)=h(r)$. Under this assumption, the complex moments of $h$ equal the moments of its arbitrary rotation $h^{\prime}$. Combining this with eq. (3) we get

$$
c_{p q}^{\left(h^{\prime}\right)}=c_{p q}^{(h)}=e^{-\mathrm{i}(p-q) \alpha} \cdot c_{p q}^{(h)} .
$$

This constraint can be fulfilled if and only if either $c_{p q}^{(h)}=0$ or $p=q$. Thus, we have shown an important property of circularly symmetric functions: If $p \neq q$ then $c_{p q}^{(h)}=0$. Consequently, eq. (4) simplifies to the form

$$
c_{p q}^{(g)}=\sum_{j=0}^{q}\left(\begin{array}{l}
p \\
k
\end{array}\right)\left(\begin{array}{l}
q \\
j
\end{array}\right) c_{j j}^{(h)} c_{p-j, q-j}^{(f)}
$$

assuming that $p \geq q$.

The assumption of circular symmetry is natural and covers many practical cases. The PSF of an out-of-focus camera is a cylinder-like function, the PSF describing longterm atmospheric turbulence blur can be approximated by a Gaussian, and the intrinsic PSF's of most optical sensors as well have this type of symmetry.

We also assume that the imaging system is energy preserving, i.e. $\iint h(x, y) d x d y \equiv c_{00}^{(h)}=1$.

\section{Invariants to convolution}

In this Section, moment invariants to convolution with a circularly symmetric PSF are introduced.

Theorem 1: Let $f(x, y)$ be an image function. Let us define for any $p>q$ functional $R^{(f)}(p, q)$ as follows:

$R(p, q)^{(f)}=c_{p q}^{(f)}-\frac{1}{c_{00}^{(f)}} \sum_{n=1}^{q}\left(\begin{array}{l}p \\ n\end{array}\right)\left(\begin{array}{l}q \\ n\end{array}\right) R(p-n, q-n)^{(f)} \cdot c_{n n}^{(f)}$

Then $R(p, q)$ is invariant to convolution, i.e. $R(p, q)^{(f * h)}=$ $R(p, q)^{(f)}$ for any circularly symmetric $h(x, y)$.

Theorem 1 can be proven by induction with respect to $q$. The invariants $R(p, q)$ are complex valued. Considering the real and imaginary parts separately we obtain two realvalued invariants. The invariants of indices $p<q$ can be defined analogously but they are useless for practical purposes because they are complex conjugated. Note that for $p=q$ there are no such invariants, because the impact of the PSF cannot be eliminated anywise.

Let us make a few notes to provide a better insight into the meaning of these convolution invariants. Any invariant (even different from those presented here) to convolution with a PSF of certain class must be constant on the images belonging to this class. This is because any image can be considered as a PSF acting on delta-function. It can be proven that if $f$ is circularly symmetric then $R(p, q)^{(f)}=0$ for any $p \neq q$. The opposite implication is valid as well. In other words, the joint null-space of all our invariants equals to the set of all circularly symmetric images. This implies the intrinsic limitation of their discriminative power - we are not able to distinguish among circularly symmetric objects. However, unlike the invariants presented in the earlier papers, the new invariants can distinguish centrosymmetric and axisymmetric shapes.

Applying Theorem 1, we can construct the invariants of any order and express them in explicit forms in terms of complex and geometric moments. The invariants up to the sixth order are shown below:

$$
\begin{array}{lll}
R(1,0)=c_{10}, & R(2,0)=c_{20}, & R(3,0)=c_{30} \\
R(4,0)=c_{40}, & R(5,0)=c_{50}, & R(6,0)=c_{60}
\end{array}
$$




$$
\begin{gathered}
R(2,1)=c_{21}-2 \frac{c_{10} c_{11}}{c_{00}}, \quad R(3,1)=c_{31}-3 \frac{c_{20} c_{11}}{c_{00}} \\
R(4,1)=c_{41}-4 \frac{c_{30} c_{11}}{c_{00}}, \quad R(5,1)=c_{51}-5 \frac{c_{40} c_{11}}{c_{00}} \\
R(3,2)=c_{32}-6 \frac{R(2,1) c_{11}}{c_{00}}-3 \frac{c_{10} c_{22}}{c_{00}} \\
R(4,2)=c_{42}-8 \frac{R(3,1) c_{11}}{c_{00}}-6 \frac{R(2,1) c_{22}}{c_{00}}
\end{gathered}
$$

\section{Invariants to convolution and rotation}

In most applications one wants to recognize objects independently of their actual orientation. However, the invariants introduced in Theorem 1 are not invariant to image rotation and cannot be directly used for this purpose. In this Section we show how to construct invariants to both convolution and rotation.

The convolution invariants from Theorem 1 have the same rotation property as the complex moments themselves, i.e.

$$
R^{\prime}(p, q)=e^{-\mathrm{i}(p-q) \alpha} \cdot R(p, q)
$$

where $\alpha$ is the angle of rotation. In other words, the magnitude does not change under rotation while the phase is shifted by $(p-q) \alpha$. We could use $|R(p, q)|$ as a convolutionrotation invariant, but the magnitudes themselves do not yield a complete system of the invariants. Better idea is to eliminate the rotation angle by multiplying several invariants of appropriate powers, which is specified in the following Theorem.

Theorem 2: Let $n \geq 1$ and let $k_{j}, p_{j}$ and $q_{j} ; j=1, \cdots, n$, be non-negative integers such that $\left(p_{j} \neq q_{j}\right)$ and $\sum_{j=1}^{n} k_{j}\left(p_{j}-q_{j}\right)=0$. Then

$$
I=\prod_{j=1}^{n} R\left(p_{j}, q_{j}\right)^{k_{j}}
$$

is invariant to rotation around the origin and to convolution with a circularly symmetric PSF.

Proof of Theorem 2 follows immediately from (7). Simple examples of the combined convolutionrotation invariants constructed according to Theorem 2 are $R(1,0) R(0,1), \quad R(1,0) R(1,2), \quad R(2,1) R(1,2)$, $R(2,1)^{3} R(0,3)$, etc.

Theorem 2 allows us to construct an infinite number of the combined invariants, but some of them are algebraically dependent on the others. They must be discarded before practical usage because they do not contribute to discrimination power at all. Theorem 2 is formally similar to the construction of pure rotation moment invariants (with no connection to convolution) from complex moments as it was presented in our recent paper [6]. One can follow that approach (using the $R(p, q)$ 's in place of $c_{p q}$ 's) to find a minimum complete and independent set of the combined invariants up to the given order.
The invariants (8) can be easily made independent on other simple transformations. Translation invariance can be achieved just by using central coordinates in the definition of complex moments. Scaling invariance can be reached via normalization of each complex moment by a factor $c_{00}^{(p+q+2) / 2}$. Invariance to the contrast changes (modelled as a multiplication of the image by a constant factor) can be achieved by normalizing each $R(p, q)$ by $c_{00}$. The same normalization helps when the assumption of preserving energy is violated. To obtain simultaneous invariance to scaling and contrast changes, one has to employ appropriate ratios of the invariants.

\section{Experiments}

Theoretical invariance of the proposed features can be in the discrete domain violated due to discretization and quantization effects and also due to the round-off errors of the calculations. The accomplished experiments tested the behavior of the invariants under discrete convolution and rotation. We also evaluated their robustness to additive random noise and their discriminative power.

First, we tested the invariance of the features defined in Theorem 1 with respect to discrete convolution. A part of Lena image sized $100 \times 100$ pixels (see Fig. 1 left) was convolved by 20 circularly symmetric masks with randomly generated diameter from 3 to 21 pixels and randomly generated non-negative coefficients (see Fig. 1 middle for an example). In each instance, all invariants $R(p, q)$ up to the sixth order along with their relative errors were calculated and the maximum relative error (MRE) was stored. All MRE's were lower than $5 \cdot 10^{-5}$ and the mean MRE was $1.03 \cdot 10^{-5}$. We repeated this experiment allowing also negative coefficients in the masks. The behavior was then slightly less stable but still very good - the mean MRE was $2.89 \cdot 10^{-5}$.

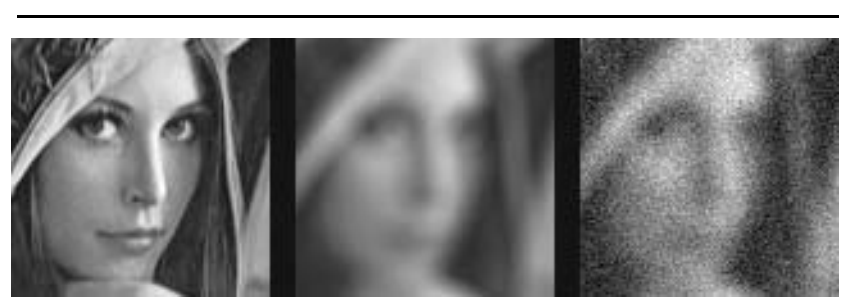

Figure 1. Examples of the test images: left original image, middle - blurred image, right blurred image with an additive noise.

In the second experiment we examined the robustness of the invariants with respect to additive noise. The experiment setup was essentially the same as in the previous case 
but each blurred image was corrupted by 100 realizations of white noise with STD varying from 0 to 40 (see Fig. 1 right). The relative error of the invariants grows slowly as the noise becomes heavy and is higher for invariants containing higher-order moments. In the presence of noise, the mask size does not have any perceptible influence on the error. For the second and third-order invariants the relative error was less than $3 \%$ while for the six-order invariants it was almost $10 \%$.

These results are not surprising and are analogous to the earlier results reported in [5] for convolution invariants with centrosymmetric PSF. This is obvious because both classes of invariants are of the same nature. We also tested the behavior of the invariants from Theorem 2 under rotation and we obtained similar results as in [5].

However, a significant difference between new and earlier invariants is in their recognition power. The invariants published in [1], [5] are invariant to broader class of centrosymmetric PSF's. Thus, they are not able to distinguish among different centrosymmetric objects. The new invariants are more specific (the PSF is assumed to have circular symmetry) and that is why they are able to distinguish centrosymmetric shapes. This may be very important when one has to recognize artificial objects (robot navigation landmarks in a complex scene for instance) because artificial objects are often symmetric. We illustrate the difference in recognition power by a simple experiment.

We tested the ability to recognize six binary shapes square, rectangle, cross, chessboard, circle, and ring, see Fig. 2 - by invariants to centrosymmetric PSF and by the new invariants to circular symmetric PSF. The results were in accordance with the theoretical-based expectation. Since all tested shapes are centrosymmetric, the values of all invariants published in [5] equal zero for all the shapes. On the other hand, the new invariants provide much better discriminability because they contain also even-order moments that are non-zero on centrosymmetric shapes (let us recall that they do not exist any even-order invariants to centrosymmetric PSF). They clearly distinguish square, chessboard, rectangle, cross, and circle. However, they are not able to distinguish between circle and ring because both shapes have circular symmetry. Detailed results are in Table 1 (only real parts of the invariants are shown, the values are appropriately normalized).

\section{Conclusion}

In this paper, the new class of moment-based features invariant to convolution with an unknown circularly symmetric PSF was introduced. They can be used for object recognition when an unknown image blurring is present. It was also shown how to achieve combined rotation-convolution invariance. Arbitrarily large systems of independent com-

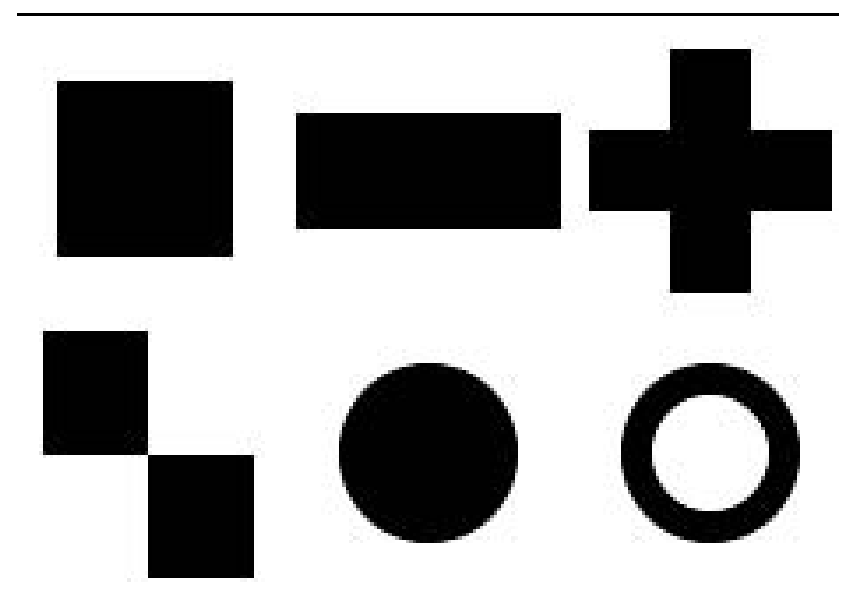

Figure 2. The test objects

\begin{tabular}{|r||r|r|r|r|c|c|}
\hline & Sq & Rect & Cross & Chess & Circ & Ring \\
\hline$R(2,0)$ & 0 & 1.21 & 0 & 2.28 & 0 & 0 \\
$R(4,0)$ & -1.54 & 1.07 & 3.97 & 3.95 & 0 & 0 \\
$R(3,1)$ & 0 & -2.93 & 0 & -10.24 & 0 & 0 \\
$R(6,0)$ & 0 & -0.53 & 0 & -3.58 & 0 & 0 \\
$R(5,1)$ & 0.70 & -0.62 & -2.73 & -4.37 & 0 & 0 \\
$R(4,2)$ & 0 & 1.90 & 0 & 12.21 & 0 & 0 \\
\hline
\end{tabular}

Table 1. The invariants for the test objects

bined invariants of any orders can be constructed in that way. Using these invariants, we avoid image deblurring and geometric normalization and, moreover, these new invariants are more discriminative and robust to noise than the blur invariants published earlier.

\section{References}

[1] J. Flusser and T. Suk, "Degraded image analysis: An invariant approach," IEEE Trans. Pattern Analysis and Machine Intelligence, vol. 20, pp. 590-603, 1998.

[2] J. Lu and Y. Yoshida, "Blurred image recognition based on phase invariants," IEICE Trans. Fundamentals of El. Comm. and Comp. Sci., vol. E82A, pp. 1450-1455, 1999.

[3] Y. Zhang, C. Wen, and Y. Zhang, "Estimation of motion parameters from blurred images," Pattern Recognition Letters, vol. 21, pp. 425-433, 2000.

[4] Y. Bentoutou, N. Taleb, M. Mezouar, M. Taleb, and L. Jetto, "An invariant approach for image registration in digital subtraction angiography," Pattern Recognition, vol. 35, pp. 2853-2865, 2002.

[5] J. Flusser and B. Zitová, "Combined invariants to linear filtering and rotation," Intl. J. Pattern Recognition Art. Intell., vol. 13, pp. 1123-1136, 1999.

[6] J. Flusser, "On the independence of rotation moment invariants," Pattern Recognition, vol. 33, pp. 1405-1410, 2000. 